\title{
Inhomogeneous Electronic Localization in Icosahedral Quasicrystals
}

\author{
A. PRekul ${ }^{a, *}$, N. Schegolikhina ${ }^{a}$ And E. Shalaeva ${ }^{b}$ \\ ${ }^{a}$ Institute of Metal Physics, Ural Department of the RAS, Ekaterinburg, Russia \\ ${ }^{b}$ Institute of Solid State Chemistry, Ural Department of the RAS, Ekaterinburg, Russia
}

\begin{abstract}
DOI: 10.12693/APhysPolA.126.556
PACS: $61.44 . \mathrm{Br}$
\end{abstract}

Experimental results indicating that the electronic inhomogeneity may be a fundamental property of icosahedral quasicrystals and their approximants are presented.

\section{Introduction}

The lattice structure of $i$-phases opened in 1984 by Shechtman [1] is based on an icosahedron - a polyhedron having symmetry of the fifth order, which cannot serve as a unit cell of a periodic crystal. It was evident from the very start that in the course of formation of thermodynamically stable $i$-phases, a minimum of free energy is possible to achieve only owing to a gain in the kinetic energy of free carriers. To this end, two mechanisms were proposed: structure-induced pseudogap in the density of states of the conduction band, which is traceable to the presence of a long aperiodic order, and electron localization, connected with either short-range order or a peculiar type of disorder.

The hypothesis of pseudogap has experimentally been shown to agree well with the estimates of electronic parameters of $i$-phases in the limit of low temperatures. These phases possess an anomalously low $\left(\approx 10^{20} \mathrm{~cm}^{-3}\right)$ concentration of conductive electrons and, consequently, anomalously low values of the metal-like conductivity, Pauli susceptibility and electronic heat capacity. The experimentally observed powerful thermal effects, namely, thermally induced charge carriers, conductivity (negative TCR), and paramagnetism, were noticed long ago to badly agree with the hypothesis of pseudogap. The width of a pseudogap $(\approx 1 \mathrm{eV})$ is too large to be the source of these effects, which meanwhile may well originate from the localized electron states with characteristic energies of excitation on the order of $\approx 10 \div 50 \mathrm{meV}$. Yet, no direct experimental evidence of such states was obtained either by high-resolution photoelectron spectroscopy or via analysis of transport properties [2]. Finally, the hypothesis of structure-induced pseudogap seemed to be a single option.

In the next years, however, several experimental observations served to revive the idea of localized states as an essential factor of the thermodynamic stability of aperiodic structures.

* corresponding author; e-mail: prekul@imp.uran.ru
First of all, these are experiments that manifested a metal-covalent nature of quasicrystals. In Refs. [3, 4], a direct evidence of covalent bonds has been proved in the electron density distribution of AlMnSi and AlReSi icosahedral quasicrystalline $1 / 1$ cubic approximants by a combination of the maximum-entropy method with the $\mathrm{Ri}-$ etveld method. In Ref. [5] a sign of covalence in AlPdRe $i$-quasicrystals has been obtained experimentally from atomic density and quasilattice constant. Based on the data obtained, a conclusion was made that localization via exchange pairing is a dominant factor of formation of the electronic structure of phases with the icosahedral short-range order and that pseudogap is a consequence of such localization.

In a number of other experiments $[6-8]$ the electronic structure of icosahedral quasicrystals $\mathrm{AlCuFe}, \mathrm{AlPdMn}$, $\mathrm{AlPdRe}$ and decagonal quasicrystals $\mathrm{d}-\mathrm{Al}-\mathrm{Ni}-\mathrm{Co}$ and their approximants was studied by means of scanning tunnel microscopy and spectroscopy. These experiments have revealed signs of a spiky local density of states near the Fermi energy with a typical energy width of 20-100 $\mathrm{meV}$. It is remarkable that spectroscopic features were shown to be of much localized nature, with a spatial extent of $0.5 \mathrm{~nm}$ and the specific electronic states to be localized on topographic features regardless of the presence of periodic or quasiperiodic long-range order $[7,8]$. These results led to a conclusion that the $\mathrm{d} I / \mathrm{d} V$ spectra are composed of two contributions: (i) a large pseudogap and (ii) a superimposed modulation consisting of small peaks and pseudogaps [8]. For the first time it was questioned [9]: What is the influence of this second characteristic of $Q C$ and approximants on the electronic transport?

Finally, in the experiments [10, 11 and refs. there] elementary electronic excitations of the $i$-phases $\mathrm{AlCuFe}$ were studied using calorimetric measurements in the temperature range $1.5-1650 \mathrm{~K}$ covering solid and liquid aggregate states of the substance. The final result for the $i$-phase $\mathrm{Al}_{63} \mathrm{Cu}_{25} \mathrm{Fe}_{12}$ is shown in Fig. 1. It turned out that, along with the linear-in-temperature contribution $\gamma T$ ( $\gamma$ is the Somerfield coefficient), which characterizes the density of states in the pseudogap, the electronic heat capacity contains a singular oscillating-in-temperature contribution whose behavior is easily traceable to two 
Schottky-like heat anomalies in the form of

$$
\begin{aligned}
C & =N_{1} k_{\mathrm{B}} \frac{\left(\frac{\delta E_{1}}{k_{\mathrm{B}} T}\right)^{2} \exp \left(\frac{\delta E_{1}}{k_{\mathrm{B}} T}\right)}{\left(1+\exp \left(\frac{\delta E_{1}}{k_{\mathrm{B}} T}\right)\right)^{2}} \\
& +N_{2} k_{\mathrm{B}} \frac{\left(\frac{\delta E_{2}}{k_{\mathrm{B}} T}\right)^{2} \exp \left(\frac{\delta E_{2}}{k_{\mathrm{B}} T}\right)}{\left(1+\exp \left(\frac{\delta E_{2}}{k_{\mathrm{B}} T}\right)\right)^{2}} .
\end{aligned}
$$

Such behavior is shown in Fig. 1 by solid lines. This contribution is insensitive to changes in the aggregate state of the substance [11]. Hence, it can be ascribed either to short-range order or disorder.

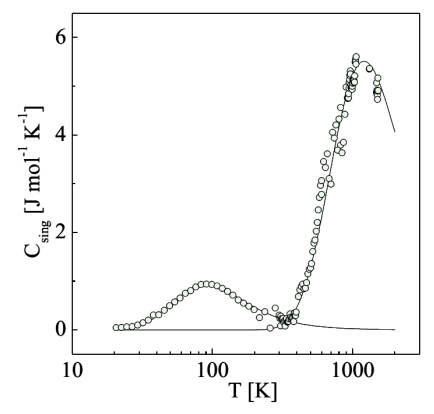

Fig. 1. Singular electronic specific heat (o) and its approximation by two Schottky-like contributions (solid lines).

Thermal Schottky anomalies, in the frame of their traditional interpretation [12], are related to two-level excitations. In such case, the above results of calorimetric experiments testify to two types/generations of localized states, each of which possesses a duplet structure with a characteristic energy of splitting of discrete/quasidiscrete levels $\delta E_{1}$ and $\delta E_{2}$, respectively.

\section{The two-component model of electronic structure and mechanism of localization}

Based on the calorimetric data described, the electronic structure of the $i$-phase $\mathrm{Al}_{63} \mathrm{Cu}_{25} \mathrm{Fe}_{12}$ can also be represented as consisting of two components: a) large pseudogap and b) two types of localized states each of which possesses a duplet structure with the Fermi level fixed in the center of each duplet. This is a significant refinement of the details of the second component, compared to [9]. In addition, it is by no means excluded that in reality there exist other generations as well, but they are not effective enough to manifest themselves in the frame of the given experiment. The picture of a formal superimposition of the components is schematically shown in Fig. 2. The presence of other possible generations is shown here by dash-and-dot line. Similarly to [9], it is reasonable to pose a question: How does each component affect physical properties of icosahedral phases?

The effect of the first component on the electronic properties of quasicrystals is studied rather well. Owing

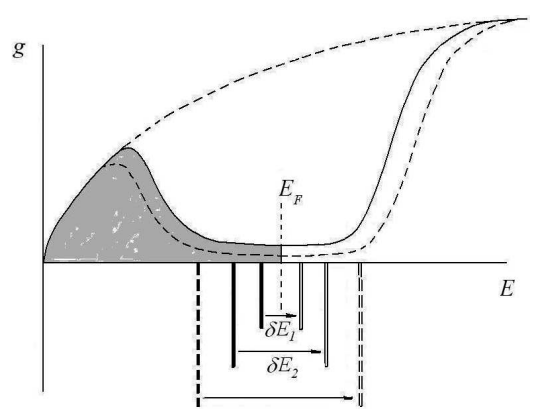

Fig. 2. Model of electron structure. The superimposition of two types of spectra - continual with pseudogap and discrete/quasidiscrete with a few types of two-level states - are shown. The Fermi level is fixed in the center of each duplet.

to this component, $i$-phases poorly differ from strongly reduced bad metals in the low-temperature limit. Generally, to pick out properties of the quasicrystals that should be ascribed to the second "discrete" spectral component is not difficult. As was already noted, the width of the pseudogap is too large to be the source of powerful thermal effects in the conductivity, magnetic susceptibility, and Hall constant. It is these effects that are of interest from the point of view of their possible dependence on the two-level excitation density, the behavior of which is determined by a sum of two contributions in the form of

$$
N=\frac{N_{1}}{1+\exp \left(\frac{\delta E_{1}}{k_{\mathrm{B}} T}\right)}+\frac{N_{2}}{1+\exp \left(\frac{\delta E_{2}}{k_{\mathrm{B}} T}\right)} .
$$

An analysis showed that to separate temperaturedependent components of the conductivity $\left(\sigma_{t}\right)$, magnetic susceptibility $\left(\chi_{t}\right.$, and Hall effect $\left(R_{\mathrm{H} t}\right)$ into individual activation contributions, similarly to the case of heat capacity, is extremely difficult, if possible at all. We had been searching for a means to show, without parceling the summed curves, that the curves contain the activationtype contributions. Let us exemplify the above with heat capacity.

The curve $C_{\text {sing }}(T)$ after integration yields the change of the internal energy of a substance $U(T)$ that comes from two-level excitations and that is a measure of the density of these excitations by definition. The curve is shown in Fig. 3. It is no difficulty to see that if the $U(T)$ curve is gained via direct measurements, it is impossible to guess that it is a sum of two contributions in the form (2). Meanwhile, an inference is easily drawn that the sign-alternating curvature of this plot is traceable to the interchange of the activation regimes. To ground this, it is sufficient to present the total curve $U(T)$ in coordinates $\ln (U)$ vs. $1 / T$ as is done in Fig. 4a. The interchange of activation regimes manifests itself in the presence of two linear portions.

Using the above approach, we analyzed the experimental curves $\sigma(T), \chi(T)$, and $R_{\mathrm{H}}(T)$ obtained earlier [13] for the $i$-phase $\mathrm{Al}_{63} \mathrm{Cu}_{25} \mathrm{Fe}_{12}$. The only fitting parameter is the value of $\sigma(0), \chi(0)$, and $R_{\mathrm{H}}(0)$, respectively. 


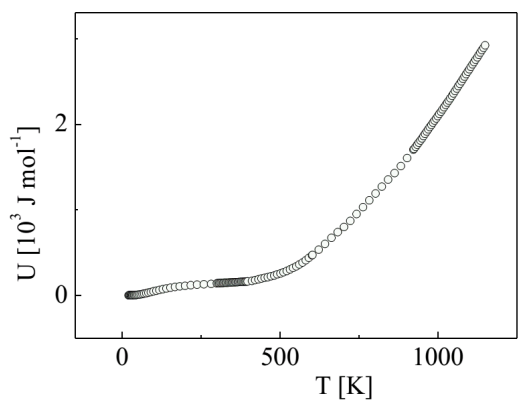

Fig. 3. Temperature dependence of the internal energy $U(T)$ that comes from two-level excitations.

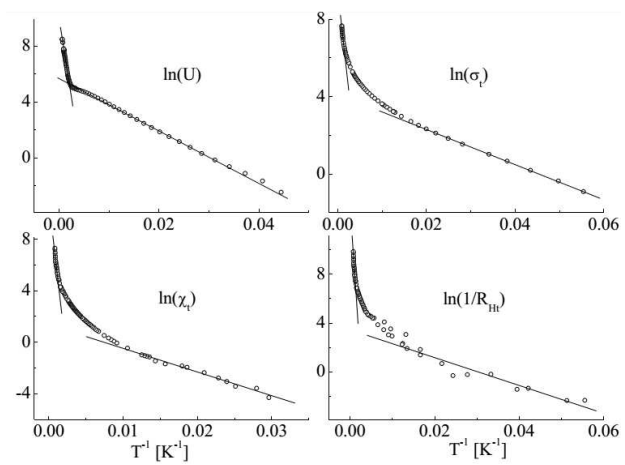

Fig. 4. Interchange of modes of thermal activation is a character feature of a two-level excitation density (a), conductivity (b), magnetic susceptibility (c) and inverse Hall effect (d) in $\mathrm{Al}_{63} \mathrm{Cu}_{25} \mathrm{Fe}_{12} i$-phase.

The results of analysis shown in Fig. 4b,c,d, hardly need explanation. All properties demonstrate behavior similar to that in Fig. 4a. The alternation of modes is quite clearly seen. Hence, all properties contain contributions that are conditioned by the temperature dependence of concentration of two-level excitations described by formula (2), and the more, are connected with the discrete component of the electronic structure.

As for the nature of the second component, judging from the above described X-ray and tunnel experiments, it is conditioned by the short-range order, which is supported by the fact that manifestations of the second component are the same both in the $i$-phases and approximants. It is interesting to verify this fact if to take the alternation of modes of thermal activation as a manifestation of the second component.

The objects of X-ray investigations in [3, 4], where direct proofs of the role of short-range order were obtained, were $i$-phases $\mathrm{Al}-\mathrm{Pd}-\mathrm{Re}$ and cubic approximants of the systems AlMnSi and AlReSi. We performed the relevant analysis of data, both ours [13] and available in literature $[14,15]$, on the electronic properties of these objects and in Figs. 5 and 6 the results are shown. The alternation of modes is quite distinct. (For the approximant AlReSi there are no data on conductivity at $T>300 \mathrm{~K}$ in the graph, therefore, the picture of changes in the ac- tivation modes is not complete.)

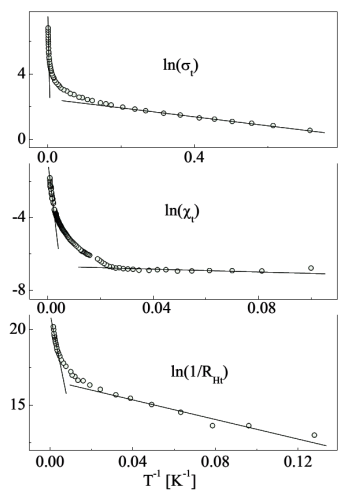

Fig. 5. Interchange of modes of thermal activation in electric, magnetic and galvanomagnetic properties of $\mathrm{Al}_{70} \mathrm{Pd}_{20} \mathrm{Re}_{10} i$-phase.

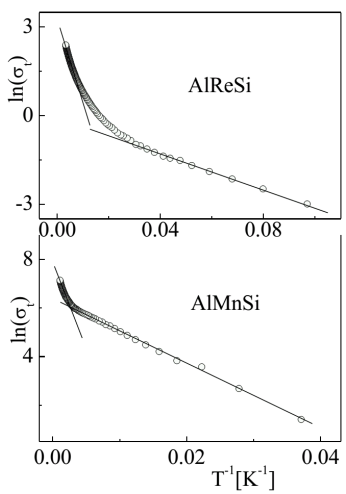

Fig. 6. Interchange of modes of thermal activation in electric properties of the cubic $1 / 1$ approximants $\mathrm{Al}_{72.4} \mathrm{Mn}_{17.5} \mathrm{Si}_{10.1}$ and $\mathrm{Al}_{72.5} \operatorname{Re}_{17.2} \mathrm{Si}_{10.1}$.

\section{Principal inference and discussion}

The latest experimental investigations have served to revive the idea of electron localization as an essential factor of the thermodynamic stability of quasicrystalline compounds. An empiric two-component model of electronic structure has arisen that includes elements of both continuous and discrete spectrum. The aim of this work was to ascertain how the discrete spectral component manifests itself in physical properties of quasicrystals. Given the detailed structure of this component, which has been obtained from the calorimetric measurements, we show that this component is responsible for the presence in quasicrystals of thermally induced charge carriers, their conductivity (negative TCR) and their paramagnetism. All these effects arise as a result of activation of electrons from the localized states possessing a duplet structure. The mechanism of localization has become clear.

Based on the above-said, it is quite easy to conclude that the discrete component of the electronic structure 
in Fig. 2 is connected with the short-range order and with localization via covalent binding. Accordingly, the two-level excitations may be transitions between symmetric/bonding and antisymmetric/antibonding states. The only question arises why the ordinary covalent bonds with so small bonding energy survive.

In conclusion we would like to draw attention of the researchers to quite unusual capabilities of the twocomponent model of electronic structure in the approximation of autonomy of its component. It is easy to notice that the localized states, if exist, coincide in energy with the continuum of residual carriers. In the frame of models of homogeneous localization such a coincidence is impossible [16]. Hence, in quasicrystals we deal with a variant of inhomogeneous electronic systems in which some types of initial valence electrons are localized whereas others are not. On the other hand, if this is the case when the localized states rather than pseudogap are electronically stabilizing factor, then thermal excitation of localized electrons into the reduced conductivity band (large pseudogap) is energetically unfavorable. It destabilizes quasilattice. Consequently, electrons come into empty/excited states of the duplet structure. Singular heat capacity and singular paramagnetism are trivial consequence of such "intra-duplet" transitions. Another case is thermally induced conductivity. We are not sure that theoretical substantiation of this fact is available in literature, so the question remains open. Take a risk on an opinion that each of the singular conductivity contributions is associated with a "thermally-induced tunneling" through the excited states of equivalent covalent bonds as shown schematically in Fig. 7.

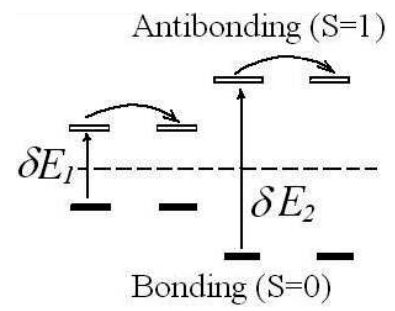

Fig. 7. Thermally-induced tunneling through the excited states of equivalent covalent bonds.

From the experimental point of view, there are no difficulties in the validity of such conductivity. If conductivity of such kind exists, it should manifest itself as an additive component to the conductivity of residual carriers $\sigma_{0}$. This model implication is supported by an empirical regularity, which has been known since the early 90ties but yet, has not been either called for or given a satisfactory explanation. We mean the so-called "Inverse Matthiessen Rule" [17]. According to this rule, the electron transport in quasicrystals is controlled by additive contributions to conductivity rather than resistivity, as in metals.

Finally, in the approximation of autonomy of components, a curious situation arises in the case of total lo- calization of initial valence electrons. The system turns out a narrow-gap semiconductor that shows up the whole bulk of the above-considered singular thermal effects. By the way, this takes place in Kondo-insulators [18, 19]. It is quite possible that the role of covalent binding in localization is not restricted to only icosahedral phases or approximants with quasicrystalline short-range order.

\section{Acknowledgments}

The authors are feeling indebted to M.I. Kurkin for valuable discussions and comments. The study was supported by the Ural Department of RAS Research Program, (project No.12-U-2-1028).

\section{References}

[1] D. Shechtman, I. Blech, D. Gratias, J.Wm. Cahn, Phys. Rev. Lett. 53, 1951 (1984).

[2] Z.M. Stadnik, in: , Physical Properties of Quasicrystals, Ed. Z.M. Stadnik, Springer Series in Solid State Physics, Springer, Berlin 1999, p. 257.

[3] K. Kirihara, T. Nakata, M. Takata, Y. Kubota, E. Nishibori, K. Kimura, M. Sakata, Phys. Rev. Lett. 85, 3468 (2000).

[4] K. Kirihara, T. Nagata, K. Kimura, K. Kato, M. Takata, E. Nishibori, M. Sakata, Phys. Rev. B 68, 014205 (2003).

[5] K. Kirihara, K. Kimura, Phys. Rev. B 64, 212201 (2001).

[6] R. Escudero, J.C. Lasjaunias, Y. Calvayrac, M. Boudard, J. Phys.: Condens. Matter 11, 383 (1999).

[7] R. Widmer, P. Gröning, M. Feuerbacher, O. Gröning, Phys. Rev. B 79, 104202 (2009).

[8] R. Mader, R. Widmer, P. Groening, P. Ruffieux, W. Steurer, O. Groening, New J. Phys. 12, 073043 (2010).

[9] R. Mader, R. Widmer, P. Groening, W. Steurer, O. Groening, Phys. Rev. B 87, 075425 (2013).

[10] A. F. Prekul, N. I. Shchegolikhina, K. Edagawa, Philos. Mag. 91, 2828 (2011).

[11] A.F. Prekul, N.I. Shchegolikhina, A.B. Gaiduchenko, K.I. Grushevskii, JETP Lett. 94, 366 (2011).

[12] W.H. Keesom, N. Pearlman, in: Handbook der Physik, Bd. 14-15, Springer-Verlag, Berlin 1956.

[13] A.F. Prekul, N.I. Schegolikhina, Crystallogr. Rep. 52, 996 (2007).

[14] C. Berger, C. Gignoux, O. Tjernberg, P. Lindqvist et al., Physica B 204, 44 (1995).

[15] R. Tamura, T. Asao, S. Takeuchi, Phys. Rev. Lett. 86, 3104 (2001).

[16] N.F. Mott, E.A. Davis, Electronic processes in noncrystalline materials, Clarendon Press, Oxford 1971; Y. Imry, Introduction to mesoscopic physics, Oxford Univ. Press, Oxford 2002.

[17] T. Klein, C. Berger, D. Mayou, F. Cyrot-Lackmann, Phys. Rev. Lett. 66, 2905 (1991).

[18] G. Aeppli, Z. Fisk, Comments Cond. Matt. Phys. 16, 155 (1992).

[19] P.S. Riseborough, Adv. Phys. 49, 257 (2000). 